(C) 2021 IEEE. Personal use of this material is permitted. Permission from IEEE must be obtained for all other uses, in any current or future media, including reprinting/republishing this material for advertising or promotional purposes, creating new collective works, for resale or redistribution to servers or lists, or reuse of any copyrighted component of this work in other works.

\title{
Understanding the Reliability of Ferroelectric Tunnel Junction Operations using an Advanced Small-Signal Model
}

\author{
Lorenzo Benatti, Francesco Maria Puglisi \\ Dipartimento di Ingegneria "Enzo Ferrari”, Via P. Vivarelli 10/1, 41125 - Modena (MO) - Italy \\ Corresponding author email: lorenzo.benatti@unimore.it phone: +39-059-2056324
}

\begin{abstract}
Ferroelectric technology is becoming ever more appealing for a variety of applications, especially analog neuromorphic computing. In this respect, elucidating the physical mechanisms occurring during device operation is of key importance to improve the reliability of ferroelectric devices. In this work, we investigate ferroelectric tunnel junctions (FTJs) consisting of a ferroelectric hafnium zirconium oxide (HZO) layer and an alumina $\left(\mathrm{Al}_{2} \mathrm{O}_{3}\right)$ layer by means of $\mathrm{C}$-f and $\mathrm{G}-\mathrm{f}$ measurements performed at multiple voltages and temperatures. For a dependable interpretation of the results, a new small signal model is introduced that goes beyond the state of the art by $i$ ) separating the role of the leakage in the two layers; $i$ i) including the significant impact of the series impedance (that depends on the samples layout); iii) including the frequency dependence of the dielectric permittivity; $i v)$ accounting for the fact that likely not the whole HZO volume crystallizes in the orthorhombic ferroelectric phase. The model correctly reproduces measurements taken on different devices in different conditions. Results highlight that the typical estimation method for interface trap density may be misleading.
\end{abstract}

Keywords - Ferroelectric Tunnel Junction, Capacitance, Small signal model, Neuromorphic.

\section{INTRODUCTION}

The need to develop energy-efficient circuits for artificial intelligence applications running on edge devices leading the exploration of innovative solutions. In this respect, the adaptive learning effect of ferroelectric materials polarization allows non-volatile multilevel memory effect [1], opening possibilities to circumvent the device- and circuit-level limitations of CMOS technology, such as difficulties in further scaling (at the device level) and the unavoidable presence of the von Neumann bottleneck (at the circuit level). Specifically, the hafnium zirconium oxide (HZO)-based Ferroelectric Tunnel Junctions (FTJs) with Metal/Insulator/Ferroelectric/Metal (MIFM) structure are promising candidates to act as synaptic elements in analog neuromorphic architectures [2]. In this respect, achieving functional circuits requires a satisfactorily large compensation of the polarization charges, that is indeed instrumental to the a)
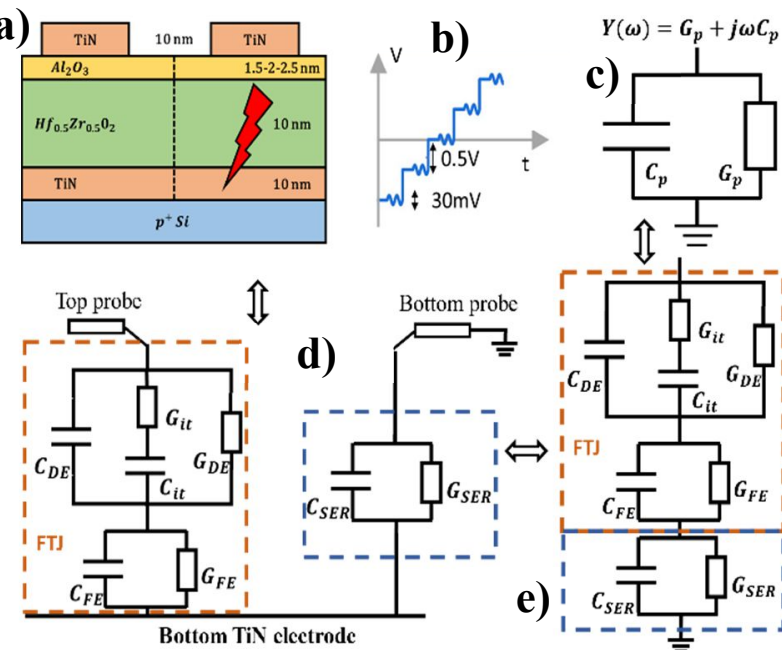

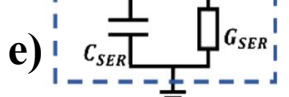

Fig. 1 - a) Layout of the FTJ stack. The access to the bottom electrode is achieved by means of a broken access device (AD), that provides connection to the bottom probe. b) C-f and G-f measurements are carried out on samples with different area and dielectric thickness. The applied DC voltage goes from $-4 \mathrm{~V}$ to $4 \mathrm{~V}$ for $t_{D E}=1.5 \mathrm{~nm},-4.5 \mathrm{~V}$ to $4.5 \mathrm{~V}$ for other thicknesses, with $0.5 \mathrm{~V}$ steps and an AC signal of $30 \mathrm{mV}$ (RMS). The objective is to evaluate the parallel capacitance and conductance (c) of the entire stack. d) Equivalent small-signal circuit of the measured sample. The AD is considered as an impedance in series with the device under investigation. The $C_{i t}-G_{i t}$ branch allows to model the equivalent trapped/interfacial charges and their $\tau$. e) Corresponding small signal model proposed in this work.

device retention. The latter is therefore intrinsically related to the dynamics of charge trapping in the defects that are present in the different layers and at the insulator/ferroelectric (IF) interface. However, the full understanding of their role and of the underlying mechanisms, critical for reliable device operation, are far from being achieved. Elucidating the physical mechanisms occurring during device operation is then of key importance to improve the reliability of ferroelectric devices. In this work, we investigate FTJs consisting of an $\mathrm{HZO}$ and alumina $\left(\mathrm{Al}_{2} \mathrm{O}_{3}\right)$ bi-layer structure by means of $\mathrm{C}-\mathrm{f}$ and $\mathrm{G}-\mathrm{f}$ measurements performed at multiple voltages and temperatures. For a dependable interpretation of the results, a new small signal model is introduced that goes 
beyond the state of the art by $i$ ) separating the role of the leakage in the two layers; $i$ ) including the significant impact of the series impedance (that depends on the samples layout); iii) including the frequency dependence of the dielectric permittivity; $i v$ ) accounting for the fact that likely not the whole HZO volume crystallizes in the orthorhombic ferroelectric phase. The model is shown to correctly reproduce measurements taken on different devices in different conditions. The paper is organized as follows: in Section II, we introduce the devices and experiments; the proposed new small-signal model is described in Section III, and its validation is shown in Section IV; in Section V, a detailed sensitivity analysis of the model parameters is
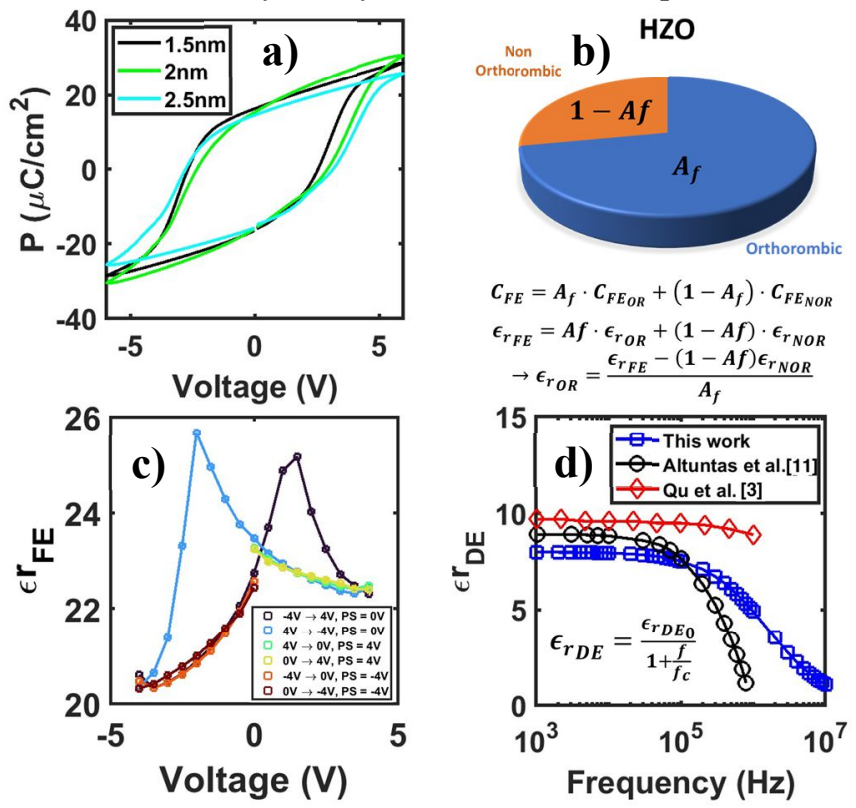

Fig. 2 - a) Polarization-voltage curves measured after wake-up on samples with different DE thickness, confirming the expected behavior. b) Since the crystallization phase of the $\mathrm{HZO}$ is not uniform, the ferroelectric is considered not completely orthorhombic. An area factor $\left(A_{f}=50-90 \%[6,7]\right)$ is then necessary to consider the contribution of this non-ideality. Equations show how the corresponding effective permittivity $\left(\epsilon_{r_{F E}}\right)$ is calculated. c) C-f G-f measurements have been carried out with different starting and pre-poling voltages. The extracted $\epsilon_{r_{F E}}$ voltage dependence matches expectation and is consistent with literature results [6,9]. The extracted values are lower than those reported for purely orthorhombic $\mathrm{HZO}$ due to $A_{f}$. d) The DE permittivity is considered frequency dependent [3,11] using the equation in the figure, in order to include the effect of the ALD. For our device, the extracted corner frequency is in the range of values already presented in the literature $[3,11]$.

reported, and its discussion is used to elucidate the role of the different layers in the measured response; in Section VI we report the results obtained by measuring and modeling different devices across the wafer, together with the results of temperature-dependent measurements. Conclusions follow.

\section{DEVICE AND EXPERIMENTS}

The FTJs analyzed in this work, fabricated by NaMLab, have a TiN $/ \mathrm{Al}_{2} \mathrm{O}_{3} / 10 \mathrm{~nm}-\mathrm{HZO} / \mathrm{TiN}$ stack, with different $\mathrm{Al}_{2} \mathrm{O}_{3}$ thicknesses $(1.5-2-2.5 \mathrm{~nm})$. The cross-sectional schematic is shown in Fig. 1a. The bottom TiN electrode (BE), the HZO (FE), and the $\mathrm{Al}_{2} \mathrm{O}_{3}$ (DE) layers were deposited by atomic layer deposition (ALD) on $\mathrm{p}^{+}$-Si wafers. Then, the top TiN electrodes (TEs) were deposited on top via a shadow mask. After deposition, all samples were annealed to crystallize the HZO film. Since the BE, common to all the devices, is not directly accessible, hard breakdown must be induced in a sacrificial device (henceforth, access device - AD) to contact the BE layer, Fig. 1a. Initially, all devices are woken up with a train of 3000 triangular pulses $( \pm 6 \mathrm{~V}, 10 \mathrm{kHz})$. After wakeup, the FTJs exhibit good ferroelectric switching and a large remnant polarization $2 P_{r} \approx 35 \mu \mathrm{C} / \mathrm{cm}^{2}$ as shown in Fig. $2 \mathrm{a}$. Then, C-f/G-f measurements (Fig. 1b) are carried out at different DC voltages ( $-4.5 \mathrm{~V}$ to $4.5 \mathrm{~V}$ for $t_{D E}=2 / 2.5 \mathrm{~nm}$ and $4 \mathrm{~V}$ to $4 \mathrm{~V}$ for $\left.t_{D E}=1.5 \mathrm{~nm}\right)$, temperatures $\left(30^{\circ} \mathrm{C}\right.$ to $\left.100^{\circ} \mathrm{C}\right)$, and frequencies (up to $10 \mathrm{MHz}$ ). The latter is an important novelty, since existing works do not explore the range above $1 \mathrm{MHz}$ $[2,3]$. The admittance of the entire stack is measured as the parallel of a capacitance $\left(\mathrm{C}_{\mathrm{p}}\right)$ and a conductance $\left(\mathrm{G}_{\mathrm{p}}\right)$, Fig. $1 \mathrm{c}$, and includes the contribution of the $\mathrm{AD}$ and of layout

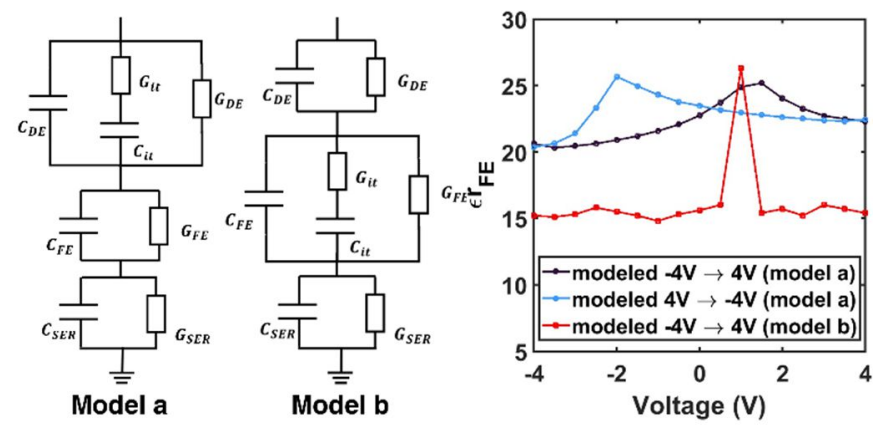

Fig. 3 - Circuit representation of the small-signal model adopted in this study (model a). A different approach, in which the $C_{i t}-G_{i t}$ branch was shifted to the FE side was explored as well (model b) but it was excluded as it is unable to reproduce the correct $\epsilon_{r_{F E}}$ voltage dependence (right panel).

parasitic, not compensated for by short-circuit compensation.

\section{Proposed SMAll-Signal Model OF THE FTJ}

To interpret the results, we introduce a small-signal model with significant novelties as compared to the existing ones in the literature $[3,4]$, see Fig. 1d-e. Specifically, $i$ ) to fully include the effect of the AD and, in general, of possible stray and interconnect impedance that is always present even in layouts in which the BE is directly accessible, we include a general series impedance, modelled as the parallel of $C_{S E R}$ and $\left.G_{S E R} ; i i\right)$ we model each layer of the FTJ by considering their capacitance and parallel conductance, the latter being representative of the leakage through that specific layer. This is different from other works $[3,4]$ in which the leakage was included by considering a global parallel conductance tied across the two layers, which did not allow to distinguish between the role played by each layer in charge transport; iii) we consider the well-known fact [5] that HZO does not crystallize fully in the orthorhombic (and ferroelectric) phase, but also exhibits non-orthorhombic grains with different permittivity values. So, we calculate the $\mathrm{FE}$ relative permittivity $\left(\epsilon_{r_{F E}}\right)$ as in Fig. $2 \mathrm{~b}$, where $\varepsilon_{O R}$ and $\varepsilon_{N O R}$ are the permittivity of the orthorhombic (i.e., taken to be around 36 [6]) and non-orthorhombic phases, respectively, and $A_{f}$ is the fraction of the area occupied by orthorhombic grains, a parameter that is then estimated by reproducing experimental data. These values are considered for simplicity to be frequency-independent, as it was shown up to a few $\mathrm{GHz}$ using dedicated test structures [8]. The $\epsilon_{r_{F E}}$ voltage dependence [6,9] is also considered. Fig. 2c shows how the model correctly converges to the expected $\epsilon_{r_{F E}}$ voltage reported in the literature $[6,9]$ without any a priori assumption, 

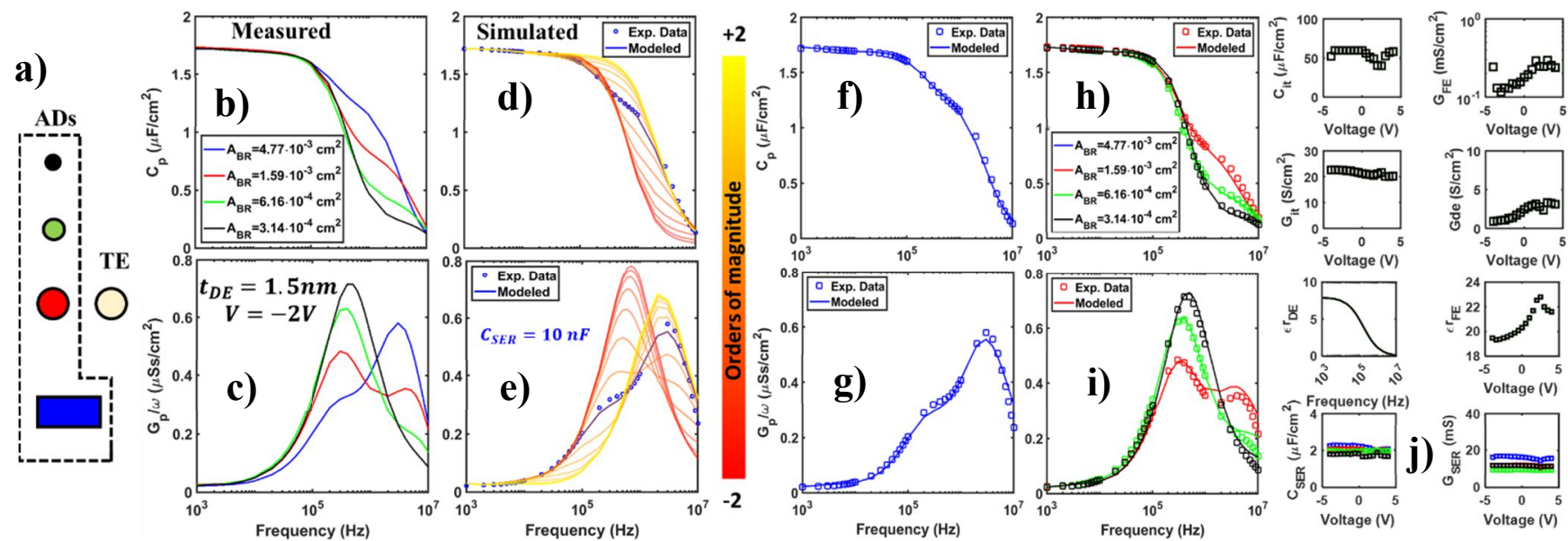

Fig. 4 - a) Representative top view of employed ADs and TE. b-c) Experimental data obtained using ADs with different areas (different $C_{S E R}-$ the same color code as in (a) is used). d-e) Measured (blue symbols) and simulated (solid lines) $C_{p}$ (d) and $G_{p} / \omega$ (e) profiles. Simulated blue lines are fitting to the experiments. Lines with different colors are derived from a sensitivity analysis on $C_{S E R}$. $C_{p}$ and $G_{p} / \omega$ profiles vary when $C_{S E R}$ is varied over \pm 2 orders of magnitude, centered on the nominal value extracted by fitting the measurements obtained by using the larger AD (blue line). All other parameters are left to the values extracted by the fitting. f-g-h-i) Modeling of data obtained using ADs with different areas (symbols with different colors - same color code as in (a) is used). Initially, measured data (symbols) in (f) and (g) are fitted at different applied voltages, resulting in the parameters reported in (j). Then, by only varying the series impedance parameters (bottom boxes in (j)) and leaving fixed all the others (boxes in (j) with only black markers or line) the $C_{p}$ and $G_{p} / \omega$ profiles corresponding to Ads with different areas are well reproduced ((h) and (i)). j) Extracted parameters. As expected, $C_{S E R}$ decreases proportionally to the AD area, as the area-normalized $C_{S E R}$ is constant, $\mathrm{AD}$-area independent, and comparable to $C_{p}$ at low frequencies. $G_{S E R} \mathrm{~S}$ are also comparable, since all $\mathrm{ADs}$ are broken with the same Ic.

which strengthens the validity of the proposed approach. To this point, several experiments have been performed to test the reliability of the model to reliably extract this parameter, carrying out $\mathrm{C}-\mathrm{f} / \mathrm{G}-\mathrm{f}$ measurements using different pre-poling and DC voltage range combinations. The perfect overlap of the values extracted at the same voltage in all measurements underlines that the values are self-consistent and validates the overall modeling approach. Furthermore, this confirms that the application of a given DC bias at each step of the C-f/G-f measurement (Fig. 1b) is sufficient to determine the number of switched ferroelectric domains even before the application of the superposed AC signal, which makes the measured frequency response free from artifacts that may come from stray polarization; $i v$ ) we include the frequency dependence of the DE permittivity using a single-pole model, see Fig. 2d, in agreement with data from the literature that clearly show how the corner frequency may be in the range explored in this work, depending on the DE thickness and deposition process. The role of the defects located both in the DE/FE layers and at the IF interface is included, as in previous works [3,4], by means of a lumped $\mathrm{C}_{\mathrm{it}}-\mathrm{G}_{\mathrm{it}}$ branch across the DE. Different attempts in positioning this branch have been done, but with ineffectual results. Fig. 3 shows that positioning the traps branch at the FE side results in a model that fails at reproducing the expected trend of $\epsilon_{r_{F E}}$ with the applied voltage, although resulting in overall satisfying fitting of the C-f and G-f measurements (not shown). Although this result is sufficient to discard this alternative model formulation, it is important to remark that, by using this approach, the extracted values of other crucial parameters are inconsistent with expectations. Specifically, the corner frequency that models the frequency dependence of $\epsilon_{r_{D E}}\left(f_{c}\right)$ is remarkably low (about $30 \mathrm{kHz}$ ), and largely below the values reported in the literature (Fig. 2d). In addition, the extracted $C_{i t}$ is lower than $1 \mu \mathrm{F} / \mathrm{cm}^{2}$, which would lead to an erroneous evaluation of the trapped charge density. Alternative small-signal models that employ a generalized leakage conductance spanning across the whole FTJ stack (instead of using dedicated a leakage conductance for each layer) have been tried, without interesting outcomes.

\section{SMALL-Signal Model VALIDATION}

To validate the model, we exploit the possibility to control the series impedance by choosing ADs with different areas (different $\mathrm{C}_{\mathrm{SER}}$ ) and break them with the same current compliance $\mathrm{I}_{\mathrm{c}}=10 \mathrm{~mA}$ (that defines $\mathrm{G}_{\mathrm{SER}}$ ). Specifically, the $\mathrm{C}_{\mathrm{p}}$ and $G_{p} / \omega$ profiles measured at $-2 \mathrm{~V}$ (i.e., the typical read voltage) on an FTJ with a $1.5 \mathrm{~nm}$ DE, using ADs with 4 different areas (sketch in Fig. 4a) are shown in Fig. 4b-c. Notably, the response significantly changes with the area of the $\mathrm{AD}$ (and thus with $\mathrm{C}_{\mathrm{SER}}$ ), underlining the significant impact of the series impedance. This is further confirmed by the sensitivity analysis carried out on $\mathrm{C}_{\mathrm{SER}}$, which clearly shows how the variation of this parameter alone has a profound impact on $C_{p}$ and $G_{p} / \omega$ profiles, in agreement with the experimental results, Fig. 4d-e. To quantify the $\mathrm{C}_{\mathrm{SER}} \mathrm{S}$ values, the model parameters were extracted by a global minimization algorithm to reproduce the data corresponding to the largest AD, blue line and symbols in Fig. 4f-g, and the extraction was repeated at all DC voltages, Fig. $4 \mathrm{j}$. Remarkably, by keeping all the parameters fixed and letting only $\mathrm{C}_{\mathrm{SER}}$ and $\mathrm{G}_{\mathrm{SER}}$ vary freely, the model can reproduce the profiles measured at different $\mathrm{AD}$ areas, Fig. 4h-i. Moreover, the extracted values of $\mathrm{C}_{\mathrm{SER}}$ (per unit area) do not change with the $\mathrm{AD}$ area and are equivalent to the value of the series of $C_{D E}$ and $C_{F E}$ (per unit area) at low frequencies. Also, the extracted $G_{S E R}$ values are similar, as the devices were all broken at the same $I_{c}$. These results validate the model ability to consider the contribution of the $\mathrm{AD}$ and, in general, of whatever series impedance may be present. This is of utter importance, since the series impedance (even when in the range of few tens of $\mathrm{pF}$ ) strongly impacts the magnitude of the peak in the $G_{p} / \omega$ profile, typically used to estimate the interface trap density $[3,4]$. Also, depending on the series impedance value, a second peak may appear above $1 \mathrm{MHz}$, 
which would go unnoticed without extending the measurement range to $10 \mathrm{MHz}$. In addition, as shown in Fig. $2 \mathrm{c}$ and $4 \mathrm{j}$, the model qualitatively reproduces the expected $\epsilon_{r_{F E}}$ voltage dependence [9], but with lower overall values as the HZO is not fully orthorhombic. Moreover, the extracted $\epsilon_{r_{D E}}$ frequency profile is compatible with literature reports $[3,11]$.

\section{SENSITIVITy ANALYSIS}

The model is now exploited to gain insights on the role played by the different layers by means of a sensitivity analysis across 4 orders of magnitude on the most relevant parameters. Results in Fig. 5 suggest that the overall leakage is most sensitive to $\mathrm{G}_{\mathrm{FE}}$, dominated by the defects in the FE layer, rather than to $\mathrm{G}_{\mathrm{DE}}$. This is an expected result, since the HZO thickness is at least 4 times larger than the respective $\mathrm{Al}_{2} \mathrm{O}_{3}$.
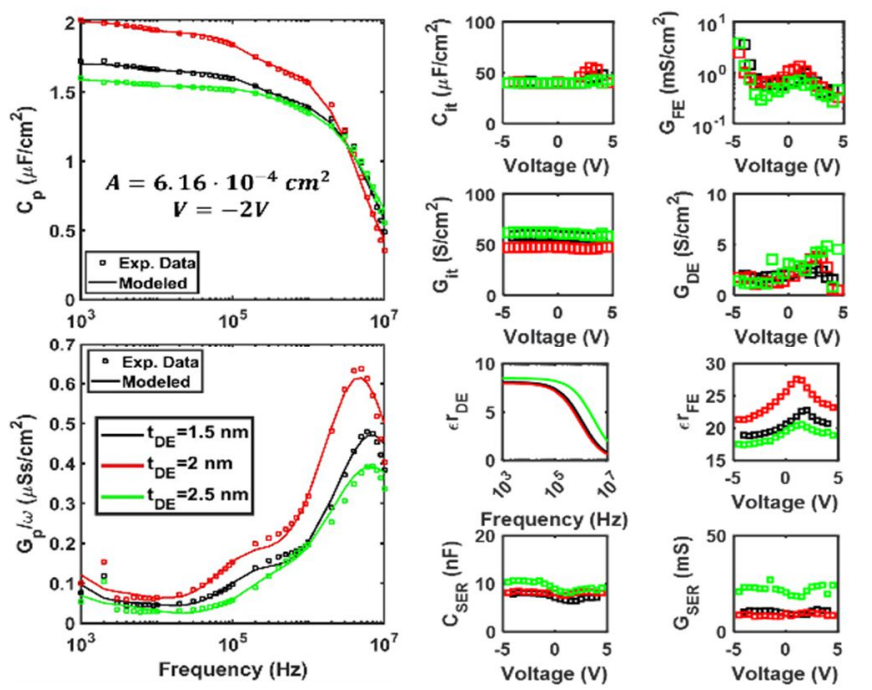

Fig. $8-C_{p}$ and $G_{p} / \omega$ profiles measured (symbols) and simulated (lines) at a DC voltage $\mathrm{V}=-2 \mathrm{~V}$ on devices with the same area and with different $t_{D E}$ (gi)ferent colors), together with the DC voltage dependence of the extracted model parameters. No trend with the dielectric thickness is visible.

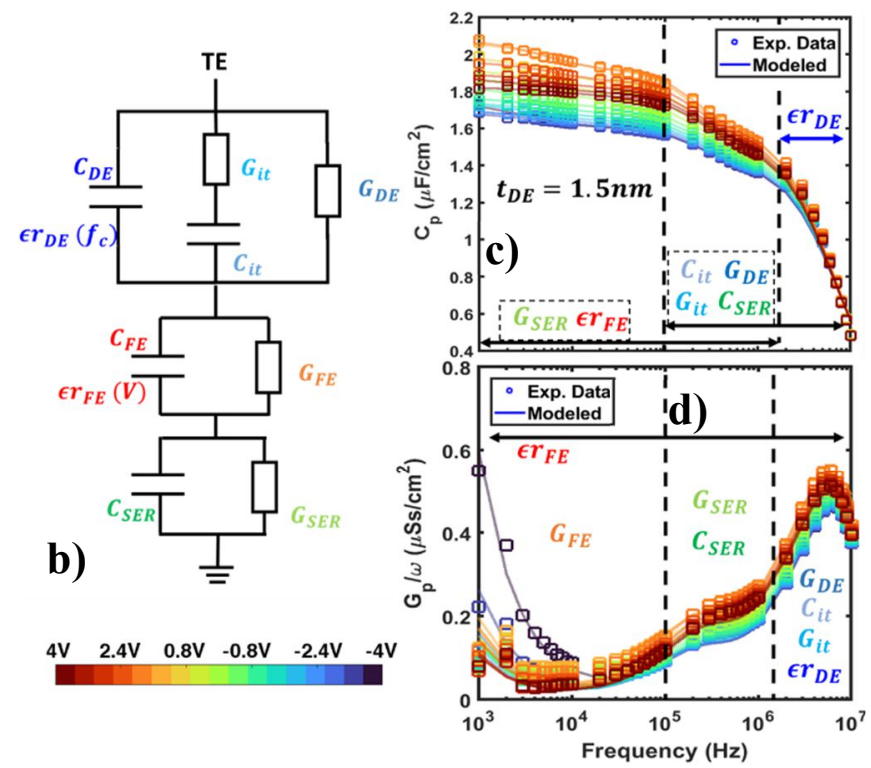

Fig. 6 - a) Employed small-signal model where each parameter is appropriately color coded. b) Color bar for measurement DC voltages applied to FTJ TE. c-d) C-f/G-f results at different DC voltages (color as in b) for a device with $t_{D E}=1.5 \mathrm{~nm}$. The region in which each parameter has the most severe impact on the curves is evidenced.

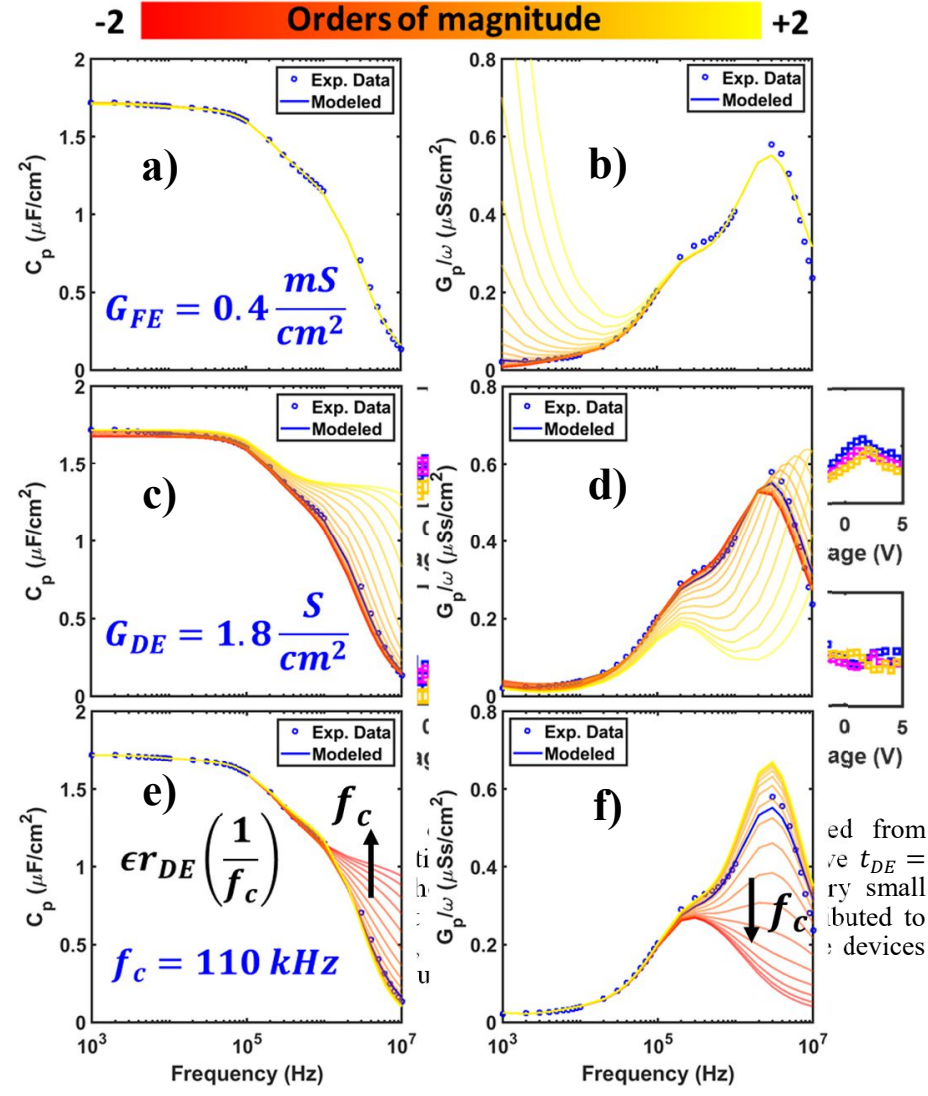

Fig. 5 - Sensitivity analysis, over \pm 2 orders of magnitude, of $C_{p}$ (a-c-e) and $G_{p} / \omega$ (b-d-f) profiles to the $\mathrm{HZO}$ conductance $\left(G_{F E}\right)$ (a-b), $\mathrm{Al}_{2} \mathrm{O}_{3}$ conductance $\left(G_{D E}\right)$ (c-d) and cutoff frequency $\left(f_{C}\right)$ of the dielectric permittivity $\left(\epsilon_{r_{D E}}\right)(\mathrm{e}-\mathrm{f})$. Blue symbols, lines, and reported values represent measured data $\left(V_{\text {Top Electrode }(T E)}=-2 \mathrm{~V}\right)$, modeling results, and corresponding extracted nominal values of the parameters vs. which the sensitivity analysis is then performed.

$\mathrm{HZO}$ acts so as a 'leakage bottleneck', and its conductance variations (hence the variation of defect density within the layer) dictates the overall leakage through the stack. The FE processing conditions and the resulting FE defectiveness have then a fundamental impact on the performance of the device, especially at low frequencies. In addition, the frequency dependence of $\epsilon_{r_{D E}}$ is shown to play a crucial role in correctly reproducing the trends above $1 \mathrm{MHz}$. In addition, it contributes in determining the magnitude of the peak in the $G_{p} / \omega$ profile, that could again perturb the estimation of the interface trap density. It is worth noting that an ideal $\mathrm{Al}_{2} \mathrm{O}_{3}$ layer $\left(f_{c} \rightarrow \infty\right)$ would suppress the parasitic effects, leading $\mathrm{G}_{\mathrm{p}} / \omega$ profile with a single peak and to a saturation of $C_{p}$ at high frequencies (instead of a roll-off). Fig. 6 summarizes the regions of the $C_{p}$ and $\mathrm{G}_{\mathrm{p}} / \omega$ profiles in which each model parameter has a more marked influence than the others. It is interesting to notice that in both profiles the low frequency behavior is mostly dominated by the HZO properties, while the high frequency response derives from a non-trivial combination of the DE leakage, the frequency dependence of the DE permittivity, the trapped charge, and the series impedance contribution. 

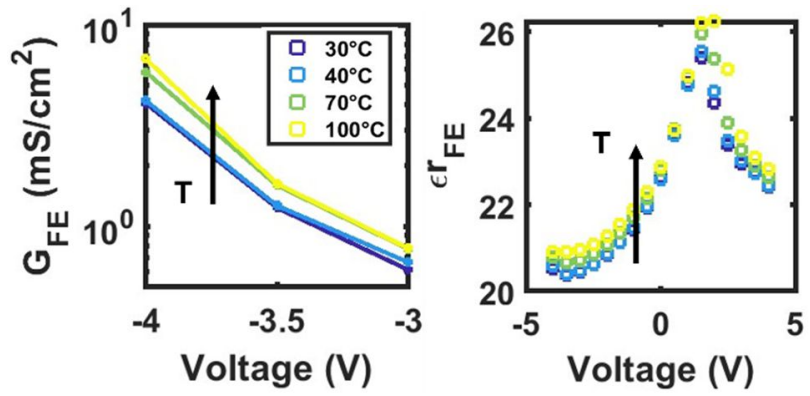

Fig. 9 - HZO parameters extracted from C-f/G-f measurements carried out at different temperatures. As expected, the permittivity of the ferroelectric increases with the temperature, as well as the HZO leakage measured at strongly negative voltages. The device has $t_{D E}=1.5 \mathrm{~nm}, A=6.16$. $10^{-4} \mathrm{~cm}^{2}$.

\section{RESULTS AND DisCUSSION}

Measurements are repeated on different samples to validate the model stability and estimate device-level variability. As shown in Fig. 7, nominally identical FTJs exhibit very small parameters dispersion. Fig. 8 shows the parameters extracted on FTJs with different DE thickness: no particular trend is evidenced, not even in $\mathrm{C}_{\mathrm{it}}$, although the $\mathrm{G}_{\mathrm{p}} / \omega$ profiles peak at different magnitudes. This underlines even further how the typical strategy to estimate the interface trap density (i.e., proportional to the peak magnitude in the $G_{p} / \omega$ profile) $[3,4]$ can lead to misleading predictions, and that a more refined modeling approach is needed. It is worth mentioning that the $\mathrm{C}_{\text {it }}$ values extracted here $\left(20-50 \mu \mathrm{F} / \mathrm{cm}^{2}\right)$ should not be rigidly interpreted as related to traps located exclusively at the IF interface (that would lead to unrealistically high densities $\approx 1$ $\left.-3 \cdot 10^{14} \mathrm{~cm}^{-2}\right)$ but rather as an average local volumetric trap density $\left(\approx 10^{20}-10^{21} \mathrm{~cm}^{-3}\right)$, since defects across almost the whole stack can respond in $\mathrm{C}-\mathrm{f} / \mathrm{G}-\mathrm{f}$ measurements, as shown by advanced multiscale simulations [12], and also play a role in compensating polarization charge. Finally, by extracting the parameters from measurements at different temperatures, we correctly reproduce the expected temperature dependence of the trap-assisted leakage in the FE (found to be close to that in $\mathrm{HfO}_{2}$ RRAMs [13]) and of the FE permittivity [14], Figs. 9-10.

\section{CONCLUSIONS}

We introduced and validated an advanced FTJ small-signal model that accounts for separate leakage contributions in the FE and DE layers, frequency dependence of DE permittivity, series impedance, and non-uniform crystalline FE phase. The model correctly reproduces measurements taken on different devices in different conditions, allowing a more refined investigation on sample layout and material properties effects on the entire device. Results highlight that the typically adopted estimation methods for interface trap density may be misleading.

\section{ACKNOWLEDGMENTS}

The authors would like to express gratitude to NaMLab for providing the devices. The work is funded by the H2020 BeFerroSynaptic (GA 871737) project. The content reflects the authors' results, but not necessarily the opinion of the EC.
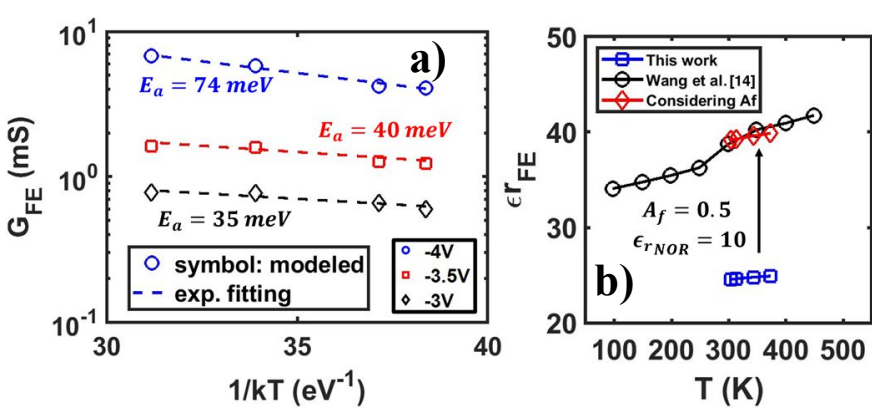

Fig. 10 - a) Arrhenius plot of FE conductance at strongly negative voltages, extracted from the data in Fig. 9. b) FE permittivity temperature dependence (blue), and its projection considering $A_{f}$ (red), that is considering a fully orthorhombic layer. Data taken at $0 \mathrm{~V}$ bias. For reference, data from the literature [14] is also reported (black).

\section{REFERENCES}

[1] S. Oh, H. Hwang, and I. K. Yoo, "Ferroelectric materials for neuromorphic computing", APL Materials 7, 091109 (2019) https://doi.org/10.1063/1.5108562

[2] Ryu, H., Wu, H., Rao, F. et al. Ferroelectric Tunneling Junctions Based on Aluminum Oxide/ Zirconium-Doped Hafnium Oxide for Neuromorphic Computing. Sci Rep 9, $20383 \quad$ (2019). https://doi.org/10.1038/s41598-019-56816-x

[3] Y. Qu, J. Li, M. Si, X. Lyu and P. D. Ye, "Quantitative Characterization of Interface Traps in Ferroelectric/Dielectric Stack Using Conductance Method," in IEEE Transactions on Electron Devices, vol. 67, no. 12, pp. 5315-5321, Dec. 2020, doi: 10.1109/TED.2020.3034564.

[4] J. Li, Y. Qu, M. Si, X. Lyu and P. D. Ye, "Multi-Probe Characterization of Ferroelectric/Dielectric Interface by $\mathrm{C}-\mathrm{V}, \mathrm{P}-\mathrm{V}$ and Conductance Methods," 2020 IEEE Symposium on VLSI Technology, 2020, pp. 1-2, doi: 10.1109/VLSITechnology18217.2020.9265069.

[5] Lun Xu, Tomonori Nishimura, Shigehisa Shibayama, Takeaki Yajima, Shinji Migita, and Akira Toriumi, "Kinetic pathway of the ferroelectric phase formation in doped $\mathrm{HfO}_{2}$ films" , Journal of Applied Physics 122, 124104 (2017) https://doi.org/10.1063/1.5003918

[6] Abdulazhanov, Sukhrob \& Le, Quang Huy \& Huynh, Dang Khoa \& Wang, Defu \& Kämpfe, Thomas. (2019). A mmWave Phase Shifter Based on Ferroelectric Hafnium Zirconium Oxide Varactors. 10.1109/IMWS-AMP.2019.8880144.

[7] M. -Y. Kao et al., "Variation Caused by Spatial Distribution of Dielectric and Ferroelectric Grains in a Negative Capacitance Field-Effect Transistor," in IEEE Transactions on Electron Devices, vol. 65, no. 10, pp. 4652-4658, Oct. 2018, doi: 10.1109/TED.2018.2864971.

[8] B. Lin, G. Choe, J. Hur, A. I. Khan, S. Yu and H. Wang, "Experimental RF Characterization of Ferroelectric Hafnium Zirconium Oxide Material at GHz for Microwave Applications," 2021 Device Research Conference (DRC), 2021, pp. 1-2, doi: 10.1109/DRC52342.2021.9467202.

[9] S. J. Kim, J. Mohan, C. D. Young, L. Colombo, J. Kim, "Ferroelectric TiN/Hf0.5Zr0.5O2/TiN Capacitors with Low-Voltage Operation and High Reliability for Next-Generation FRAM Applications," 2018 IEEE International Memory Workshop (IMW), 2018, pp. 1-4, doi: 10.1109/IMW.2018.8388832.

[10] Guo, Yao \& Wei, Xianlong \& Shu, Jiapei \& Liu, Bo \& Yin, Jianbo \& Guan, Changrong \& Yuxiang, Han \& Gao, Song \& Chen, Qing. (2015). Charge trapping at the MoS2-SiO2 interface and its effects on the characteristics of MoS2 metal-oxide-semiconductor field effect transistors. Applied Physics Letters. 106. 103109. 10.1063/1.4914968.

[11] Halit Altuntas, Kemal Kaplan, Electrical conduction mechanisms and dielectric relaxation in $\mathrm{A} 12 \mathrm{O} 3$ thin films deposited by thermal atomic layer deposition, Materials Science in Semiconductor Processing, Volume 86, 2018, Pages 111-114, ISSN 1369-8001, https://doi.org/10.1016/j.mssp.2018.06.027.

[12]G. Sereni, L. Vandelli, D. Veksler and L. Larcher, "A New Physical Method Based on CV - GV Simulations for the Characterization of the Interfacial and Bulk Defect Density in High- k /III-V MOSFETs," in IEEE Transactions on Electron Devices, vol. 62, no. 3, pp. 705-712, March 2015, doi: 10.1109/TED.2014.2385959.

[13]F. M. Puglisi, A. Qafa and P. Pavan, "Temperature Impact on the Reset Operation in $\mathrm{HfO}_{2}$ RRAM," in IEEE Electron Device Letters, vol. 36, no. 3, pp. 244-246, March 2015, doi: 10.1109/LED.2015.2397192. 
[14]D. Wang, J. Wang, Q. Li, W. He, M. Guo, A. Zhang, Z. Fan, D. Chen, M. Qin, M. Zeng, "Stable ferroelectric properties of $\mathrm{Hf}_{0.5} \mathrm{Zr}_{0.5} \mathrm{O}_{2}$ thin films within a broad working temperature range" 2019 Jpn. J. Appl. Phys. 58 090910. doi.org/10.7567/ 1347$4065 / \mathrm{ab} 3844$ 